\title{
Competition of van der Waals and chemical forces on gold-sulfur surfaces and nanoparticles
}

Jeffrey R. Reimers ${ }^{1,2}$, Michael J. Ford ${ }^{2}$, Sebastian M. Marcuccio ${ }^{3,4}$, Jens Ulstrup ${ }^{5}$, and Noel S. Hush ${ }^{6,7}$

${ }^{1}$ International Centre for Quantum and Molecular Structures, College of Sciences, Shanghai University, Shanghai 200444, China.

${ }^{2}$ School of Mathematical and Physical Sciences, The University of Technology Sydney, Sydney NSW 2007 Australia.

${ }^{3}$ Department of Chemistry and Physics, La Trobe Institute for Molecular Science, La Trobe University, Melbourne, Victoria 3086, Australia.

${ }^{4}$ Advanced Molecular Technologies Pty Ltd, Unit 1, 7-11 Rocco Drive Scoresby, Vic. 3179, Australia.

${ }^{5}$ Department of Chemistry, Technical University of Denmark, Kongens Lyngby 2800, Denmark.

${ }^{6}$ School of Chemistry F11, The University of Sydney, NSW 2006 Australia.

${ }^{7}$ School of Molecular Bioscience, The University of Sydney, NSW 2006 Australia.

Nature Reviews Chemistry 1, 0017 (2017)

Errors existed in the originally published version of figure 5 in this Review. Thiolate RS ${ }^{-}$, generated from thiol RSH and $\mathrm{NaBH}_{4}$, reacts with $\mathrm{HAuCl}_{2}$ to afford $\mathrm{Au}(\mathrm{i})$ thiolates. $\mathrm{Au}(0)$ thiyls can form on treating Au metal with RSH or RSSR, or from a mixture containing $\mathrm{HAuCl}_{2}$, RSSR and acid. The amended figure 5 appears in both the HTML and PDF versions. 